Article

\title{
On the Design of an Elegant CFO Estimate System with the Assistance of Pilots for OFDM Transmission
}

Tsui-Tsai Lin ${ }^{1}$ and Fuh-Hsin Hwang ${ }^{2, *}$

${ }^{1}$ Department of Electronics Engineering, National United University, No. 2 Lien-Da, Miaoli 360, Taiwan; E-Mail: ttlincs@nuu.edu.tw

${ }^{2}$ Department of Optoelectronics and Communication Engineering,

National Kaohsiung Normal University, No. 62, Shenjhong Road, Yanchao, Kaohsiung 824, Taiwan

* Author to whom correspondence should be addressed; E-Mail: fuhsin.h@gmail.com;

Tel.: +886-7717-2930 (ext. 7716); Fax: +886-7605-1146.

Academic Editor: Hung-Yu Wang

Received: 6 October 2015 / Accepted: 8 December 2015 / Published: 12 December 2015

\begin{abstract}
We present an improved line search method of the carrier frequency offset (CFO) estimate technique for OFDM systems aided with pilots. Unlike the conventional approaches, prior knowledge of channel order is unnecessary for the presented scheme, because we apply an interpolation technique for obtaining the extended channel frequency response. The search mechanism of the proposed approach is primarily based on the criterion that CFO can be found by discovering the frequency that achieves the minimum value of the well-defined channel residual energy. In addition, we substitute the line search method with an iterative approach to reduce the proposed estimator's complexity. Analytical and simulation results have been conducted to verify the efficacy of the proposed schemes in this paper.
\end{abstract}

Keywords: orthogonal frequency division multiplexing (OFDM); carrier frequency offset (CFO); pilot-based CFO estimation; interpolation; iterative

\section{Introduction}

In wireless communications, the carrier frequency offset (CFO) [1,2] is primarily caused when the frequencies of the oscillators between the transmitting and receiving ends do not match or there is a Doppler frequency shift that is proportional to the carrier frequency, as well as to the moving speed 
of the mobile unit. For orthogonal frequency division multiplexing (OFDM) systems [3,4], the quality of the signal transmission is liable to degrade in the case of the existence of CFO. This is because CFO destroys the orthogonality between the subcarriers and, thus, brings about the signal distortion in OFDM systems. Since multi-carrier systems are very sensitive to the subcarrier synchronization error, it is essential to provide an accurate estimation for compensating the CFO. Otherwise, it is difficult to develop an OFDM system that is able to meet the elementary requirement of acceptable communication link quality. Therefore, CFO estimation for OFDM attracts much attention and is an active area of research.

In the past, a number of works (e.g., [1-17]) have been devoted to the CFO estimation techniques. The least square (LS) criterion is used in [8] for CFO estimation. Hebley [7] presented a CFO estimator based on the maximum-likelihood (ML) criterion while assuming the training sequences and channel statistics are known. In [9], the ML criterion is further employed for the joint estimation of channel and CFO. In [1,10,11], CFO estimators were developed using a training sequence with two or multiple identical components. By using one OFDM symbol with $M$ identical components, the CFO estimation scheme in [11] expands the estimation range to $M$ subcarrier spacing for reducing the implementation cost, since the wider the estimation range is, the lower the precision of the employed oscillator will be. However, the expense of the scheme [11] is the increase of the computational complexity, especially for a large $M$. The estimation range can be further expanded by using one more OFDM symbol [12] while sacrificing the transmission efficiency. The CFO estimation algorithms with the cyclic prefix (CP) technique [13], which either use a special training sequence [14] or a pilot signal [15], have been presented. One of the most commonly used is to employ the subspace estimation algorithm of the singular value decomposition (SVD) [16] and the ML criterion [17]. On the other hand, it is commonly assumed that the channel side information is unavailable to the CFO estimator, and CFO estimation can also be reached by employing the inherent structure of OFDM signals. This is generally categorized into the field of blind approaches. For instance, the ML-based CFO estimator is implemented by using correlation operations, as the training signals are periodic and unknown $[1,10]$. The blind CFO estimation has received a great amount of attention because of its bandwidth efficiency. However, the blind schemes degrade in performance significantly, especially when the length of the channel impulse response is large. In practice, many channel estimation and space-time coding schemes [18] require the channel statistics information. For these systems, the availability of channel statistics information can be expected for CFO estimation in OFDM.

The objective of this paper is to present a CFO estimation technique, which is aided with pilot symbols, for OFDM transmission. The results obtained from this novel approach would provide two major contributions. For the traditional line search methods of CFO, it is often assumed that the channel order is known prior to the CFO estimate. However, this assumption may not be realistic, while the channel characteristic is difficult to be acquired promptly. First, we discard the assumption and modified the traditional $\mathrm{CFO}$ line search method by introducing an interpolation technique to attempt reconstructing full-dimensional channel frequency responses for an OFDM system with pilots. We derived the theoretical analysis in this study and reached some beneficial statistics of the estimation scheme. Based on these obtained analytical results, it can be found that the CFO estimate criterion is equivalent to discovering the frequency that achieves the minimum residual channel energy (CRE). 
Secondarily, we efficiently reduced the implementation complexity of the proposed CFO estimator by means of developing a simplified scheme without using the exhaustive CFO search method. Such a scheme allows the estimator to need less amount of computation during the data process than that required by a conventional line search approach. In spite of providing a lower complexity than that of a conventional technique, the alternative scheme operates at the expense of a little amount of estimate accuracy loss. Therefore, we further applied an iteration technique to compensate the estimate accuracy loss, which results from the necessary approximation in the analytical process of the estimate statistics.

The organization of the rest of the paper is as follows. Section 2 depicts the mathematical models for the received data while considering the presence of CFO and provides an essential review of the conventional estimate approach presented by Chung [19]. In Section 3, we first propose an improved search-based CFO estimator while assuming the channel order is not reached. Next, an iterative algorithm is presented to reduce the computational complexity of the proposed estimator. To have a clear insight of how much complexity can be saved, numerical comparison of the computational complexities among all of the concerned schemes is also included in this section. In Section 4, we provide the computer simulation results to demonstrate the performance advantages of the proposed schemes over Chung's approach. Finally, the conclusion is reported in Section 5.

The notations used in this paper are defined as followed: vectors (matrices) are typed with boldface letters. $\mathbf{I}_{n}, \mathbf{O}_{m \times n}$ and $\mathbf{0}_{n}$ represent an $n \times n$ identity matrix, an $m \times n$ all-zero matrix and an $n \times 1$ all-zero vector, respectively. $\mathbf{D}_{\mathbf{a}}$ is a diagonal matrix with the diagonal entries taken from the vector $\mathbf{a} .(\cdot)^{*},(\cdot)^{T}$, $(\cdot)^{H}$ and $\operatorname{Tr}\{\cdot\}$ the complex conjugate, transpose, Hermitian transpose and trace operations, respectively. The $(i, j)$-th entry of matrix $\mathbf{A}$ is denoted by $[\mathbf{A}]_{i j}$. In addition, $\Re\{\cdot\}, E\{\cdot\}$ and $\|\cdot\|$ denote the real part of a complex number, the taken ensemble average and the Frobenius norm operator, respectively.

\section{Signal Model}

For a classical OFDM system, we consider that it has a total bandwidth composed of $N$ subcarriers and employs the technique of pilot-based signal correction, in which quadrature amplitude modulation (QAM) transmission is utilized over multipath fading channels. Let the total $K$ pilot symbols and $(N-K)$ data symbols be modulated each by an individual subcarrier, such that the inter-carrier interference is absent. The modulated symbols $\mathrm{X}$ are processed by the inverse fast Fourier transformer (IFFT), and then transformed into the time-domain signals obtained by $\mathbf{x}=\mathbf{F}^{H} \mathbf{X}$, where $\mathbf{F} \in C^{N \times N}$ denotes the FFT matrix with the $(k, l)$-th entry being $e^{j 2 \pi(k-1)(l-1) / N} / \sqrt{N}$. For a channel of maximum delay spread $L$, the OFDM symbol requires a cyclic prefix (CP) of length $N_{g} \geq L$ so as to eliminate the inter-symbol interference (ISI). After the $\mathrm{CP}$ is removed at the receiver end, the received OFDM symbols are then FFT processed. In the presence of CFO, the received symbol block is given by:

$$
\begin{aligned}
\mathbf{R} & =\mathbf{F D}_{\mathbf{a}\left(\epsilon_{d}\right)} \mathbf{F}^{H} \mathbf{D}_{\mathbf{X}} \mathbf{F h}+\mathbf{W} \\
& =\mathbf{F D}_{\mathbf{a}\left(\epsilon_{d}\right)} \mathbf{F}^{H} \mathbf{D}_{\mathbf{X}} \mathbf{H}+\mathbf{W}
\end{aligned}
$$

where $\epsilon_{d}$ is the related frequency offset normalized to subcarrier spacing and the diagonal matrix $\mathbf{D}_{\mathbf{a}\left(\epsilon_{d}\right)}$ represents the CFO effect with $\mathbf{a}\left(\epsilon_{d}\right)=\left[1, e^{j 2 \pi \epsilon_{d} / N}, \cdots, e^{j 2(N-1) \pi \epsilon_{d} / N}\right]^{T}$. The channel impulse response is given by $\mathbf{h}=\left[h(0), h(1), \cdots, h(L), \mathbf{0}_{N-L-1}^{T}\right]^{T}$, and the $N \times 1$ vector $\mathbf{H}=\mathbf{F h}$ is the channel 
frequency response (CFR). W denotes the complex white Gaussian channel noise, which is assumed to be independent of the transmitted data.

The channel estimation (CE) aims at extracting $\mathbf{H} / \mathbf{h}$ from the received data in Equation (1). We briefly review the pilot-based CE technique, which is widely used because of its simplicity. Let the $k$-th pilot symbol be modulated on the $c_{k}$-th subcarrier where $k=0,1, \ldots, K-1$. Stacking all of the $K$ entries in order yields a $K \times 1$ vector $\mathbf{R}_{P}$, which is expressed by:

$$
\begin{aligned}
\mathbf{R}_{P} & \triangleq\left[\begin{array}{lll}
R_{c_{0}} & R_{c_{1}} \cdots & R_{c_{K-1}}
\end{array}\right]^{T}=\mathbf{S R} \\
& =\mathbf{S F D}_{\mathbf{a}\left(\epsilon_{d}\right)} \mathbf{F}^{H} \mathbf{D}_{\mathbf{X}} \mathbf{H}+\mathbf{S W}
\end{aligned}
$$

where the $K \times N$ matrix $\mathbf{S}=\left[\mathbf{e}_{c_{0}}, \mathbf{e}_{c_{1}}, \cdots, \mathbf{e}_{c_{K-1}}\right]^{T}$, with $\mathbf{e}_{k}$ being the $k$-th column vector of an $N \times N$ identity matrix $\mathbf{I}_{N}$. Based on the pre-known pilot symbols, the CFR estimate associated with the pilot symbols can be written by:

$$
\begin{aligned}
\hat{\mathbf{H}}_{P} & =\mathbf{D}_{\mathbf{X}_{P}}^{-1} \mathbf{R}_{P} \\
& =\mathbf{D}_{\mathbf{X}_{P}}^{-1} \mathbf{S F D} \mathbf{D}_{\mathbf{a}\left(\epsilon_{d}\right)} \mathbf{F}^{H} \mathbf{D}_{\mathbf{X}} \mathbf{H}+\mathbf{D}_{\mathbf{X}_{P}}^{-1} \mathbf{S W}
\end{aligned}
$$

where $\mathbf{X}_{P}=\mathbf{S X}$ is the data vector associated with the corresponding pilot symbols. Clearly, when CFO is absent $\left(\epsilon_{d}=0\right)$, we have $\mathbf{D}_{\mathbf{a}\left(\epsilon_{d}\right)}=\mathbf{I}_{N}$, which allows the CFR to be successfully estimated. When the input signal-to-noise ratio (SNR) is sufficiently large, the estimated CFR reaches:

$$
\begin{aligned}
\hat{\mathbf{H}}_{P} & =\mathbf{S H}+\mathbf{D}_{\mathbf{X}_{P}}^{-1} \mathbf{S W} \\
& \approx \mathbf{S H}
\end{aligned}
$$

However, in mobile environments, CFO usually exists due to Doppler shift and/or mismatch between the transmitter and receiver oscillators. Even though the CFO is very small, the estimator provided by Equation (3) may not work properly. To relieve the impact on the system performance caused by CFO, a variety of approaches have been proposed in the past. Let us consider a typical example, which employs redundant pilot symbols to perform CFO estimation [19]. Recall that the desired channel response with a channel length of $L$ can be modeled by $\mathbf{h}=\left[h(0), h(1), \cdots, h(L) \mathbf{0}_{N-L-1}^{T}\right]^{T}$, where $h(i) \neq 0$, $i=0,1, . ., L$ and the other entries are all zeros. For the estimated channel response $\hat{\mathbf{h}}$, the last $N-L-1$ entries of $\hat{\mathbf{h}}$ are negligible, because they are essentially induced by the channel noise while CFO is absent. Else, the magnitudes of these entries increase with the value of CFO. That is, the minimization of the energy composed of the last $N-L-1$ entries of $\hat{\mathbf{h}}$ can be regarded as a useful criterion for estimating the CFO. The procedure of Chung's algorithm [19] is summarized as follows:

(1) Compute the CFO-compensated data:

$$
\tilde{\mathbf{R}}(\epsilon)=\mathbf{F D}_{\mathbf{a}(\epsilon)}^{H} \mathbf{F}^{H} \mathbf{R}
$$

(2) Compute the CFR estimate of pilot symbols according to Equation (3):

$$
\hat{\mathbf{H}}_{P}(\epsilon)=\mathbf{D}_{\mathbf{X}_{P}}^{-1} \mathbf{S} \tilde{\mathbf{R}}(\epsilon)
$$

(3) By operating $K$-point inverse discrete Fourier transform (IDFT), the time-domain channel response estimate of size $K$ is given by:

$$
\begin{aligned}
\hat{\mathbf{h}}_{\text {Chung }}(\epsilon) & =\mathbf{S} \mathbf{F}^{H} \mathbf{S}^{T} \hat{\mathbf{H}}_{P}(\epsilon) \\
& =\mathbf{S} \mathbf{F}^{H} \mathbf{S}^{T} \mathbf{D}_{\mathbf{X}_{P}}^{-1} \mathbf{S} \tilde{\mathbf{R}}(\epsilon)
\end{aligned}
$$


(4) Compute the energy of the last $K-L-1$ entries of $\hat{\mathbf{h}}_{\text {Chung }}(\epsilon)$, which is termed the channel residual energy (CRE), defined by:

$$
\begin{aligned}
f(\epsilon) & =\sum_{j=\hat{L}+2}^{K}\left|\left[\hat{\mathbf{h}}_{\text {Chung }}(\epsilon)\right]_{j}\right|^{2} \\
& =\left|\operatorname{Jh}_{\text {Chung }}(\epsilon)\right|^{2}
\end{aligned}
$$

where $\mathbf{J}=\left[\mathbf{O}_{(K-\hat{L}-1) \times(\hat{L}+1)}, \mathbf{I}_{K-\hat{L}-1}\right]$ and $\hat{L}$ is the estimate of the channel order $L$.

(5) Determine the CFO estimate in accordance with:

$$
\begin{aligned}
\hat{\epsilon}_{d} & =\arg \min _{\epsilon} \quad f(\epsilon) \\
& =\arg \min _{\epsilon}\left\|\mathbf{J F}_{K}^{H} \mathbf{D}_{\mathbf{X}_{P}}^{-1} \mathbf{S} \tilde{\mathbf{R}}(\epsilon)\right\|^{2}
\end{aligned}
$$

where $\mathbf{F}_{K}$ denotes the first $K$ columns of $\mathbf{F}$.

In spite of proving good performance, Chung's method deteriorates in performance while $L$ is not correctly estimated. Under the condition of $\hat{L}>L$, although the last few entries of $\hat{\mathbf{h}}_{\text {Chung }}(\epsilon)$ may be very small, they result in estimation errors. On the other hand, this scheme fails to provide acceptable performance when $\hat{L}<L$. How to correctly estimate the channel order is a critical challenge for Chung's algorithm. What is worse is that this method breaks down as the number of pilot symbols, $K$, is less than or equal to the channel order. In addition, the searching granularity for $\epsilon_{d}$ in Equation (9) is given by a heuristic approach, which involves the trade-off of estimate accuracy and computational complexity. For these considerations, in the next section, we will present a robust CFO estimator for a pilot-based OFDM system while avoiding these unfavorable factors.

\section{Robust Low-Complexity CFO Estimator}

In this section, we develop a robust CFO estimator whose computational complexity is greatly improved. Especially, the proposed CFO estimator is able to promise a reliable performance without knowledge of the channel order $L$. To this end, we apply the CP length, $N_{g}$, to compute the CRE instead of $L$. In spite of avoiding dealing with the estimation of $L$, the proposed CFO estimator cannot work properly in a channel with a long delay spread. This is because that large channel order results in the degree-of-freedom deficiency problem while computing the CRE. Fortunately, here, we present the reconstruction of a full-dimensional CFR of size $N$ by imposing the interpolation technique on the CFR of the pilot symbols.

\subsection{Proposed Search-Based CFO Estimator}

Let $\mathbf{Q}$ denote the $N \times K$ interpolation matrix. The linear interpolation is widely used, and for the considered system, the $N \times K$ matrix $\mathbf{Q}$ is given by:

$$
\mathbf{Q}=\left[\begin{array}{ccccc}
\mathbf{q}_{1} & \mathbf{q}_{2} & & & \\
& \mathbf{q}_{1} & \mathbf{q}_{2} & & \\
& & \ddots & \ddots & \\
& & & \mathbf{q}_{1} & \mathbf{q}_{2} \\
& & & & \mathbf{q}_{1}
\end{array}\right]
$$


where:

$$
\begin{aligned}
& \mathbf{q}_{1}=\frac{1}{N} \times[N, N-K, N-2 K, \cdots, 2 K, K]^{T} \\
& \mathbf{q}_{2}=\frac{1}{N} \times[0, K, 2 K, \cdots, N-2 K, N-K]^{T}
\end{aligned}
$$

The full-dimensional CFR can be derived from Equation (3) and written by the expression:

$$
\begin{aligned}
\hat{\mathbf{H}}(\epsilon) & =\mathbf{Q} \hat{\mathbf{H}}_{P}(\epsilon) \\
& =\mathbf{Q D}_{\mathbf{X}_{P}}^{-1} \mathbf{S} \tilde{\mathbf{R}}(\epsilon)
\end{aligned}
$$

Applying $N$-point IFFT to $\hat{\mathbf{h}}$, the full-dimensional time-domain channel response reaches:

$$
\begin{aligned}
\hat{\mathbf{h}}(\epsilon) & =\mathbf{F}^{H} \hat{\mathbf{H}}(\epsilon) \\
& =\mathbf{F}^{H} \mathbf{Q} \mathbf{D}_{\mathbf{X}_{P}}^{-1} \mathbf{S} \tilde{\mathbf{R}}(\epsilon) .
\end{aligned}
$$

Summing up the energy of the last $N-N_{g}$ entries of $\hat{\mathbf{h}}(\epsilon)$ in Equation (13), the CRE is given by:

$$
\begin{aligned}
f(\epsilon) & =\sum_{j=N_{g}+1}^{N}\left|[\hat{\mathbf{h}}(\epsilon)]_{j}\right|^{2} \\
& =\left\|\tilde{\mathbf{J}} \mathbf{F}^{H} \mathbf{Q D}_{\mathbf{X}_{P}}^{-1} \mathbf{S F D}_{\mathbf{a}(\epsilon)}^{H} \mathbf{r}\right\|^{2} \\
& =\mathbf{a}^{T}(\epsilon) \mathbf{D}_{\mathbf{r}}^{H} \mathbf{F}^{H} \mathbf{S}^{T} \mathbf{D}_{\mathbf{X}_{P}^{*}}^{-1} \mathbf{Q}^{H} \mathbf{F} \tilde{\mathbf{J}}^{T} \tilde{\mathbf{J}} \mathbf{F}^{H} \mathbf{Q D}_{\mathbf{X}_{P}}^{-1} \mathbf{S F D}_{\mathbf{r}}^{T} \mathbf{a}^{*}(\epsilon) \\
& =\mathbf{a}^{H}(\epsilon) \mathbf{D}_{\mathbf{r}} \mathbf{T}^{*} \mathbf{D}_{\mathbf{r}}^{H} \mathbf{a}(\epsilon)
\end{aligned}
$$

where:

$$
\begin{aligned}
& \tilde{\mathbf{J}}=\left[\mathbf{O}_{\left(N-N_{g}\right) \times N_{g}}, \mathbf{I}_{N-N_{g}}\right] \\
& \mathbf{T}=\mathbf{F}^{H} \mathbf{S}^{T} \mathbf{D}_{\mathbf{X}_{P}^{*}}^{-1} \mathbf{Q}^{H} \mathbf{F} \tilde{\mathbf{J}}^{T} \tilde{\mathbf{J}} \mathbf{F}^{H} \mathbf{Q D}_{\mathbf{X}_{P}}^{-1} \mathbf{S F} \\
& \mathbf{r}=\mathbf{F}^{H} \mathbf{R} \\
& \mathbf{D}_{\mathbf{X}_{P}}^{H}=\mathbf{D}_{\mathbf{X}_{\mathbf{P}}^{*}}
\end{aligned}
$$

Note that the last equation in Equation (14) holds since the CRE is a positive real number. Clearly, the value of the expression Equation (14) achieves a minimum value when $\epsilon=\epsilon_{d}$. Thus, the CFO estimate can be determined in accordance with the criterion shown below:

$$
\begin{aligned}
\hat{\epsilon}_{d} & =\arg \min _{\epsilon} \quad f(\epsilon) \\
& \equiv \arg \min _{\epsilon} \quad \mathbf{a}^{H}(\epsilon) \mathbf{D}_{\mathbf{r}} \mathbf{T}^{*} \mathbf{D}_{\mathbf{r}}^{H} \mathbf{a}(\epsilon)
\end{aligned}
$$

From Equation (16), the choice of the granularity, $\Delta_{\epsilon}$, involves the tradeoff between the estimator's performance and computational complexity. That is, decreasing $\Delta_{\epsilon}$ can improve the estimator's performance at the expense of additional computational complexity, which is proportional to $\Delta_{\epsilon}^{-1}+1$.

\subsection{Performance Analysis}

Since $f(\epsilon)$ is a function of the normalized frequency $\epsilon$ and the proposed criterion for determining the CFO estimate is based on the minimization, which is expressed by Equation (16), the proposed criterion is similar to that of the minimum variance distortionless response (MVDR) spectrum estimator [20]. Specifically, the CFO estimate $\hat{\epsilon}_{d}$ satisfies the condition:

$$
\dot{f}\left(\hat{\epsilon}_{d}\right)=\left.\frac{d}{d \epsilon} f(\epsilon)\right|_{\epsilon=\hat{\epsilon}_{d}}=0
$$


Let $\Delta \epsilon=\hat{\epsilon}_{d}-\epsilon_{d}$ denote the error (bias) of the proposed estimator. Expanding the first-order Taylor series of $\dot{f}(\epsilon)$ around $\epsilon_{d}$, the approximation expression of $\dot{f}\left(\hat{\epsilon}_{d}\right)$ can be expressed as follows:

$$
\begin{aligned}
\dot{f}\left(\hat{\epsilon}_{d}\right) & \approx \dot{f}\left(\epsilon_{d}\right)+\ddot{f}\left(\epsilon_{d}\right) \Delta \epsilon_{d} \\
& =2 \times \Re\left\{\dot{\mathbf{a}}^{H}\left(\epsilon_{d}\right) \mathbf{D}_{\mathbf{r}} \mathbf{T}^{*} \mathbf{D}_{\mathbf{r}}^{H} \mathbf{a}\left(\epsilon_{d}\right)\right\} \\
& +2 \times \Re\left\{\dot{\mathbf{a}}^{H}\left(\epsilon_{d}\right) \mathbf{D}_{\mathbf{r}} \mathbf{T}^{*} \mathbf{D}_{\mathbf{r}}^{H} \dot{\mathbf{a}}\left(\epsilon_{d}\right)+\mathbf{a}^{H}\left(\epsilon_{d}\right) \mathbf{D}_{\mathbf{r}} \mathbf{T}^{*} \mathbf{D}_{\mathbf{r}}^{H} \ddot{\mathbf{a}}\left(\epsilon_{d}\right)\right\} \Delta \epsilon_{d}
\end{aligned}
$$

where:

$$
\begin{aligned}
& \dot{f}\left(\epsilon_{d}\right)=2 \times \Re\left\{\dot{\mathbf{a}}^{H}\left(\epsilon_{d}\right) \mathbf{D}_{\mathbf{r}} \mathbf{T}^{*} \mathbf{D}_{\mathbf{r}}^{H} \mathbf{a}\left(\epsilon_{d}\right)\right\} \\
& \ddot{f}\left(\epsilon_{d}\right)=2 \times \Re\left\{\dot{\mathbf{a}}^{H}\left(\epsilon_{d}\right) \mathbf{D}_{\mathbf{r}} \mathbf{T}^{*} \mathbf{D}_{\mathbf{r}}^{H} \dot{\mathbf{a}}\left(\epsilon_{d}\right)+\mathbf{a}^{H}\left(\epsilon_{d}\right) \mathbf{D}_{\mathbf{r}} \mathbf{T}^{*} \mathbf{D}_{\mathbf{r}}^{H} \ddot{\mathbf{a}}\left(\epsilon_{d}\right)\right\} \\
& \dot{\mathbf{a}}\left(\epsilon_{d}\right)=\left.\frac{d}{d \epsilon} \mathbf{a}(\epsilon)\right|_{\epsilon=\epsilon_{d}} \\
& \ddot{\mathbf{a}}\left(\epsilon_{d}\right)=\left.\frac{d^{2}}{d \epsilon^{2}} \mathbf{a}(\epsilon)\right|_{\epsilon=\epsilon_{d}}
\end{aligned}
$$

Furthermore, substituting the following equalities:

$$
\begin{aligned}
\mathbf{D}_{\mathbf{r}} \mathbf{a}^{*}\left(\epsilon_{d}\right) & =\mathbf{F}^{H} \mathbf{D}_{\mathbf{X}} \mathbf{H}+\mathbf{v} \\
& =\mathbf{s}+\mathbf{v} \\
\mathbf{D}_{\mathbf{r}} \dot{\mathbf{a}}^{*}\left(\epsilon_{d}\right) & =-\mathbf{D}_{\dot{\mathbf{a}}_{0}}(\mathbf{s}+\mathbf{v}) \\
\mathbf{D}_{\mathbf{r}} \ddot{\mathbf{a}}^{*}\left(\epsilon_{d}\right) & =\mathbf{D}_{\ddot{\mathbf{a}}_{0}}(\mathbf{s}+\mathbf{v})
\end{aligned}
$$

into Equation (18) yields the first and second derivatives of $f\left(\epsilon_{d}\right)$ in the form:

$$
\begin{aligned}
\dot{f}\left(\epsilon_{d}\right) & =(\mathbf{s}+\mathbf{v})^{H}\left(\mathbf{D}_{\dot{\mathbf{a}}_{0}} \mathbf{T}-\mathbf{T} \mathbf{D}_{\dot{\mathbf{a}}_{0}}\right)(\mathbf{s}+\mathbf{v}) \\
& =(\mathbf{s}+\mathbf{v})^{H} \mathbf{A}(\mathbf{s}+\mathbf{v}) \\
\ddot{f}\left(\epsilon_{d}\right) & =(\mathbf{s}+\mathbf{v})^{H}\left[-2 \mathbf{D}_{\dot{\mathbf{a}}_{0}} \mathbf{T} \mathbf{D}_{\dot{\mathbf{a}}_{0}}+\mathbf{T} \mathbf{D}_{\ddot{\mathbf{a}}_{0}}+\mathbf{D}_{\ddot{\mathbf{a}}_{0}} \mathbf{T}\right](\mathbf{s}+\mathbf{v}) \\
& =(\mathbf{s}+\mathbf{v})^{H} \boldsymbol{\Gamma}(\mathbf{s}+\mathbf{v})
\end{aligned}
$$

where, by definition,

$$
\begin{aligned}
& \mathbf{s}=\mathbf{F}^{H} \mathbf{D}_{\mathbf{X}} \mathbf{H}=\mathbf{F}^{H} \mathbf{D}_{\mathbf{H}} \mathbf{X}, \\
& \mathbf{v}=\mathbf{D}_{\mathbf{w}} \mathbf{a}^{*}\left(\epsilon_{d}\right) \\
& \mathbf{w}=\mathbf{F}^{H} \mathbf{W} \\
& \dot{\mathbf{a}}_{0}=\left.\dot{\mathbf{a}}(\epsilon)\right|_{\epsilon=0} \\
& \ddot{\mathbf{a}}_{0}=\left.\ddot{\mathbf{a}}(\epsilon)\right|_{\epsilon=0} \\
& \mathbf{A}=\mathbf{D}_{\dot{\mathbf{a}}_{0}} \mathbf{T}-\mathbf{T D}_{\dot{\mathbf{a}}_{0}} \\
& \boldsymbol{\Gamma}=-2 \mathbf{D}_{\dot{\mathbf{a}}_{0}} \mathbf{T} \mathbf{D}_{\dot{\mathbf{a}}_{0}}+\mathbf{T} \mathbf{D}_{\ddot{\mathbf{a}}_{0}}+\mathbf{D}_{\ddot{\mathbf{a}}_{0}} \mathbf{T}
\end{aligned}
$$

Recall that the estimate $\hat{\epsilon}_{d}$ is reached by minimizing the CRE, i.e., $\dot{f}\left(\hat{\epsilon}_{d}\right)=0$. Hence, the estimation error $\Delta \epsilon_{d}$ can be expressed by:

$$
\ddot{f}\left(\epsilon_{d}\right) \Delta \epsilon_{d}=-\dot{f}\left(\epsilon_{d}\right)
$$

It is not convenient to solve Equation (23) straightforwardly, because $\ddot{f}\left(\epsilon_{d}\right)$ depends on the received signals. For sufficiently high SNR, the mean value of $\ddot{f}\left(\epsilon_{d}\right)$ is much larger than the root mean square fluctuation of $\Delta \epsilon_{d}$, and we can reach a good approximation by replacing it with its expected value obtained by [11]:

$$
E\left\{\ddot{f}\left(\epsilon_{d}\right)\right\}=E\left\{(\mathbf{s}+\mathbf{v})^{H} \boldsymbol{\Gamma}(\mathbf{s}+\mathbf{v})\right\}
$$


Since the noise vector $\mathbf{v}$ is statistically equivalent to $\mathbf{w}$, it follows that $E\{\mathbf{v}\}=\mathbf{0}$ and $E\left\{\mathbf{v} \mathbf{v}^{H}\right\}=\sigma_{n}^{2} \mathbf{I}_{N}$. Hence, Equation (24) can be rewritten as:

$$
\begin{aligned}
E\left\{\ddot{f}\left(\epsilon_{d}\right)\right\} & =\mathbf{s}^{H} \boldsymbol{\Gamma} \mathbf{s}+\sigma_{n}^{2} \operatorname{Tr}\{\boldsymbol{\Gamma}\} \\
& =\mathbf{s}^{H} \boldsymbol{\Gamma} \mathbf{s} \\
& =\mu
\end{aligned}
$$

where, by definition, $\mu=\mathbf{s}^{H} \mathbf{\Gamma}$. According to Equations (23) and (25), the estimation error (bias) is easily shown as:

$$
\begin{aligned}
\Delta \epsilon_{d} & \approx \frac{\dot{f}\left(\epsilon_{d}\right)}{E\left\{\dot{f}\left(\epsilon_{d}\right)\right\}} \\
& =\mu^{-1}(\mathbf{s}+\mathbf{v})^{H} \mathbf{A}(\mathbf{s}+\mathbf{v})
\end{aligned}
$$

By taking expectation on the estimation error shown in Equation (26), we obtain the result:

$$
\begin{aligned}
E\left\{\Delta \epsilon_{d}\right\} & \approx \mu^{-1} \mathbf{s}^{H} \mathbf{A} \mathbf{s}+\mu^{-1} E\left\{\mathbf{v}^{H} \mathbf{A} \mathbf{v}\right\} \\
& =\mu^{-1} \mathbf{s}^{H} \mathbf{A} \mathbf{s}+\mu^{-1} \sigma_{n}^{2} \operatorname{Tr}\{\mathbf{A}\} \\
& =\alpha \mu^{-1}
\end{aligned}
$$

where $\alpha=\mathbf{s}^{H}$ As. Using the fact of the Gaussian noise $\mathbf{v}$ and discarding the self-product term of the noise, the mean square error is further expressed as:

$$
\begin{aligned}
E\left\{\left(\Delta \epsilon_{d}\right)^{2}\right\} & \approx \mu^{-2}\left\{\alpha^{2}+2 \alpha E\left\{\mathbf{v}^{H} \mathbf{A} \mathbf{v}\right\}+E\left\{\left(\mathbf{s}^{H} \mathbf{A} \mathbf{v}+\mathbf{v}^{H} \mathbf{A} \mathbf{s}\right)^{2}\right\}\right\} \\
& =\mu^{-2}\left(\alpha^{2}+2 \alpha \sigma_{n}^{2} \operatorname{Tr}\{\mathbf{A}\}+\sigma_{n}^{2} \mathbf{s}^{H} \mathbf{A} \mathbf{A} \mathbf{s}\right)
\end{aligned}
$$

For high SNR, Equation (28) reduces to:

$$
E\left\{\left(\Delta \epsilon_{d}\right)^{2}\right\} \approx \mu^{-2} \alpha^{2}
$$

\subsection{Iterative CFO Estimator}

Even though the proposed technique is shown to provide a reliable CFO estimate effectively, how to reduce the implementation complexity is a real challenge, especially for an OFDM system that has a large number of subcarriers. Here, we propose an efficient method to further improve the computational complexity. In comparison with the traditional approaches, the presented scheme applies the Taylor series expansion technique to simplify the optimum problem instead of using exhaustive search for finding the minimum value in Equation (14). For a small $\epsilon$, the CFO-driven diagonal matrix $\mathbf{D}_{\mathbf{a}(\epsilon)}$ can be approximated to a first-order Taylor series expansion expressed by:

$$
\mathbf{D}_{\mathbf{a}(\epsilon)} \approx \mathbf{I}_{N}+\epsilon \mathbf{D}_{\dot{\mathbf{a}}_{0}}
$$

Substituting Equation (30) into Equation (13), we have:

$$
\hat{\mathbf{h}}(\epsilon) \approx \mathbf{F}^{H} \mathbf{Q D}_{\mathbf{X}_{P}}^{-1} \mathbf{S}\left(\mathbf{I}_{N}+\epsilon \mathbf{F} \mathbf{D}_{\dot{\mathbf{a}}_{0}} \mathbf{F}^{H}\right) \mathbf{R}
$$

According to Equation (31), we can approximate the CRE in Equation (14) as:

$$
\begin{aligned}
f(\epsilon) & \approx\left\|\tilde{\mathbf{J}} \mathbf{F}^{H} \mathbf{Q} \mathbf{D}_{\mathbf{X}_{P}}^{-1} \mathbf{S}\left(\mathbf{I}_{N}+\epsilon \mathbf{F} \mathbf{D}_{\dot{\mathbf{a}}_{0}} \mathbf{F}^{H}\right) \mathbf{R}\right\|^{2} \\
& =\mathbf{r}^{H} \mathbf{T r}+\epsilon \mathbf{r}^{H} \mathbf{A r}-\epsilon^{2} \mathbf{r}^{H} \mathbf{D}_{\dot{\mathbf{a}}_{0}} \mathbf{T} \mathbf{D}_{\dot{\mathbf{a}}_{0}} \mathbf{r}
\end{aligned}
$$


The approximation in Equation (32) reveals that the optimum problem is simplified to a one-dimension optimization by minimizing the CRE value. Clearly, it can be easily shown that the optimal selection of $\hat{\epsilon}_{d}$ is derived as:

$$
\hat{\epsilon}_{d}=\frac{\mathbf{r}^{H} \mathbf{A r}}{2 \times \mathbf{r}^{H} \mathbf{D}_{\dot{\mathbf{a}}_{0}} \mathbf{T} \mathbf{D}_{\dot{\mathbf{a}}_{0}} \mathbf{r}}
$$

Because the $N \times N$ matrix $\mathbf{T}$ is independent of the received data, it can be pre-determined while performing the $\mathrm{CFO}$ estimation.

The above-mentioned method only works on the premise that $\mathbf{D}_{\mathbf{a}(\epsilon)}$ is well approximated to $\left(\mathbf{I}_{N}+\epsilon \mathbf{D}_{\dot{\mathbf{a}}_{0}}\right)$ if CFO is small enough, i.e., $\epsilon_{d} \ll 1$. However, when $\epsilon_{d}$ does not satisfy the condition of $\epsilon_{d} \ll 1$, Equation (30) does not hold any more, and thus, we cannot obtain a reliable CFO estimate by means of Equation (33). For this reason, it is important to find an alternative approach for enhancing the robustness against CFO when $\epsilon_{d}$ is not small. Next, an iteration scheme is proposed instead. Specifically, we use the current CFO estimate in Equation (33) to compensate for the received data in the next iteration. Repeating the iterations and using the $(j-1)$-th iteration result, denoted as $\hat{\epsilon}_{d}^{(j-1)}$, along with linear algebraic manipulations, the CFO estimate at the $j$-th iteration is expressed as:

$$
\begin{aligned}
\hat{\epsilon}_{d}^{(j)} & =\frac{\mathbf{r}^{(j-1) H} \mathbf{A r}^{(j-1)}}{2 \times \mathbf{r}^{(j-1) H} \mathbf{D}_{\mathbf{a}_{0}} \mathbf{T} \mathbf{D}_{\dot{\mathbf{a}}_{0}} \mathbf{r}^{(j-1)}} \\
& =\frac{\mathbf{r}^{H} \boldsymbol{\Lambda}\left(\epsilon_{d}^{(j-1)}\right) \mathbf{A} \boldsymbol{\Lambda}^{H}\left(\epsilon_{d}^{(j-1)}\right) \mathbf{r}}{2 \times \mathbf{r}^{H} \boldsymbol{\Lambda}\left(\epsilon_{d}^{(j-1)}\right) \mathbf{D}_{\dot{\mathbf{a}}_{0}} \mathbf{T} \mathbf{D}_{\dot{\mathbf{a}}_{0}} \boldsymbol{\Lambda}^{H}\left(\epsilon_{d}^{(j-1)}\right) \mathbf{r}}
\end{aligned}
$$

where, by definition,

$$
\begin{aligned}
& \boldsymbol{\Lambda}\left(\epsilon_{d}^{(j)}\right)=\left.\mathbf{D}_{\mathbf{a}(\epsilon)}\right|_{\epsilon=\hat{\epsilon}_{d}^{(j)}} \\
& \mathbf{r}^{(j)}=\boldsymbol{\Lambda}^{H}\left(\epsilon_{d}^{(j)}\right) \mathbf{r}
\end{aligned}
$$

In general, the iterative approach ultimately achieves a precision CFO estimate. However, the execution of the iterations frequently involves intensive computations. Recall that, for each iteration, the CFO estimation of our proposed scheme requires $O\left(2 N^{2}\right)$ complexity for computing $\boldsymbol{\Lambda}\left(\epsilon_{d}^{(j-1)}\right) \mathbf{D}_{\dot{\mathbf{a}}_{0}} \mathbf{T} \mathbf{D}_{\dot{\mathbf{a}}_{0}} \Lambda^{H}\left(\epsilon_{d}^{(j-1)}\right)$ and $\boldsymbol{\Lambda}\left(\epsilon_{d}^{(j-1)}\right) \mathbf{A} \boldsymbol{\Lambda}^{H}\left(\epsilon_{d}^{(j-1)}\right)$. The major disadvantage of the iterative approach is that the implementation complexity strongly depends on the CFO estimate $\hat{\epsilon}_{d}^{(j)}$. This prevents it from being used in practical applications, especially when the number of subcarriers $N$ is large. By using the commutative law of linear algebraic, we have:

$$
\mathbf{r}^{H} \boldsymbol{\Lambda}\left(\epsilon_{d}^{(j-1)}\right)=\mathbf{a}^{T}\left(\hat{\epsilon}_{d}^{(j-1)}\right) \mathbf{D}_{\mathbf{r}^{*}}
$$

Substituting Equation (36) into Equation (34), an alternative expression for $\hat{\epsilon}_{d}^{(j)}$ is rewritten by:

$$
\begin{aligned}
\hat{\epsilon}_{d}^{(j)} & =\frac{\mathbf{a}^{T}\left(\hat{\epsilon}_{d}^{(j-1)}\right) \mathbf{D}_{\mathbf{r}}^{H} \mathbf{A} \mathbf{D}_{\mathbf{r}} \mathbf{a}^{*}\left(\hat{\epsilon}_{d}^{(j-1)}\right)}{2 \times \mathbf{a}^{T}\left(\hat{\epsilon}_{d}^{(j-1)}\right) \mathbf{D}_{\mathbf{r}}^{H} \mathbf{D}_{\mathbf{a}_{0}} \mathbf{T} \mathbf{D}_{\dot{a}_{0}} \mathbf{D}_{\mathbf{r}} \mathbf{a}^{*}\left(\hat{\epsilon}_{d}^{(j-1)}\right)} \\
& =\frac{\mathbf{a}^{T}\left(\hat{\epsilon}_{d}^{(j-1)}\right) \mathbf{\Phi} \mathbf{a}^{*}\left(\hat{\epsilon}_{d}^{(j-1)}\right)}{2 \times \mathbf{a}^{T}\left(\hat{\epsilon}_{d}^{(j-1)}\right) \mathbf{\Psi} \mathbf{a}^{*}\left(\hat{\epsilon}_{d}^{(j-1)}\right)}
\end{aligned}
$$

where:

$$
\begin{aligned}
& \boldsymbol{\Phi}=\mathbf{D}_{\mathbf{r}}^{H} \mathbf{A D}_{\mathbf{r}} \\
& \boldsymbol{\Psi}=\mathbf{D}_{\mathbf{r}}^{H} \mathbf{D}_{\dot{\mathbf{a}}_{0}} \mathbf{T D}_{\dot{\mathbf{a}}_{0}} \mathbf{D}_{\mathbf{r}}
\end{aligned}
$$

Since the Hermitian matrices $\Phi$ and $\Psi$ are independent of the CFO estimate, they are computed only once during the whole iteration process. 


\subsection{Computational Complexity}

In this subsection, we investigate the computational complexity for Chung's and our proposed schemes, respectively, where the complexity is evaluated by counting the numbers of their required complex multiplications. For construction of the CFO-compensated data in Equation (5), it require $2 N^{2}+N$ complex multiplications. In addition, the calculation of $\mathbf{J F}_{K}^{H} \mathbf{D}_{\mathbf{X}_{p}}^{-1} \mathbf{S} \tilde{\mathbf{R}}(\epsilon)$ and the vector norm needs $K(K-\hat{L}-1)$ and $(K-\hat{L}-1)$ complex multiplications, respectively. As a result, the required complex multiplications for Chung's estimator using an $N_{u}$-section search are approximately expressed as:

$$
\mathrm{CM}_{\text {Chung }} \approx N_{u}[N(2 N+1)+(K-1)(K-\hat{L}-1)]
$$

As regards the proposed search-based scheme, the computation of the $\left(N-N_{g}\right) \times N$ matrix $\tilde{\mathbf{J}} \mathbf{F}^{H} \mathbf{Q D}_{\mathbf{X}_{P}} \mathbf{S F}$, which is independent of the received data, can be pre-computed and saved in a lookup-table (LUT) ROM. Thus, the proposed search-based estimator only requires $N\left(N-N_{g}\right)$ complex multiplications for computing $\tilde{\mathbf{J}} \mathbf{F}^{H} \mathbf{Q D}_{\mathbf{X}_{P}} \mathbf{S F D}_{\mathbf{r}}$. In addition, the calculation of CRE in Equation (14) needs $(N+1)\left(N-N_{g}\right)$ complex multiplications for each of $\epsilon$. Given an $N_{u}$, the total number of complex multiplications for the proposed search-based estimator is approximately:

$$
\mathrm{CM}_{\text {Search-based }} \approx\left(N-N_{g}\right)\left[N\left(N_{u}+1\right)+N_{u}\right]
$$

To reach the $j$-th CFO estimate in Equation (37), the construction of the $N \times N$ matrices $\boldsymbol{\Phi}$ and $\Psi$ requires $2 N^{2}$ complex multiplications. In addition, we require $N^{2}+N$ complex multiplications to compute either $\mathbf{a}^{T}\left(\hat{\epsilon}_{d}^{j-1}\right) \boldsymbol{\Phi} \mathbf{a}^{*}\left(\hat{\epsilon}_{d}^{j-1}\right)$ or $\mathbf{a}^{T}\left(\hat{\epsilon}_{d}^{j-1}\right) \mathbf{\Psi} \mathbf{a}^{*}\left(\hat{\epsilon}_{d}^{j-1}\right)$. Consequently, the total number of complex multiplications for the proposed iterative estimator is about:

$$
\mathrm{CM}_{\text {Iterative }} \approx 2 N[N(J+2)+J]
$$

Table 1 lists the computational complexity for the three CFO estimation approaches. Since $N_{u} \gg 1$, the proposed search-based estimation requires much lower computational complexity than Chung's scheme. Besides, inasmuch as $J \ll N$, it is shown that the proposed iterative estimator significantly outperforms both Chung's and the proposed search-based schemes in computational complexity.

\begin{tabular}{|c|c|}
\hline Algorithm & Numbers of Complexity Multiplications (CMs) \\
\hline \multirow{2}{*}{ Chung's estimator in Equation (9) } & $N_{u}[N(2 N+1)+(K-1)(K-\hat{L}-1)]$ \\
\hline & $\approx 2 N_{u} N^{2}, \quad N \gg 1$ \\
\hline Proposed search-based estimator in Equation (16) & $\begin{array}{l}\left(N-N_{g}\right)\left[N\left(N_{u}+1\right)+N_{u}\right] \\
\quad \approx N N_{u}\left(N-N_{g}\right), \quad N \gg 1\end{array}$ \\
\hline Proposed iterative estimator in Equation (37) & $\begin{array}{l}2 N[N(J+2)+J] \\
\quad \approx 2 N^{2}(J+2)\end{array}$ \\
\hline
\end{tabular}

Table 1. Comparison for the computational complexities among the proposed and conventional schemes. 


\section{Computer Simulations}

In this section, we provide computer simulations to verify the efficacy of the proposed CFO estimate technique. Consider an OFDM system with 256 subcarriers $(N=256)$ that employs QPSK modulation and a CP of length $64\left(N_{g}=N / 4=64\right)$ over a multipath fading channel. For the simplicity of implementation, here, the 64 pilot symbols $(K=64)$ are equally interlaced with an interval of four-subcarrier spacing in the span of frequency. The characteristic of the background channel is described as the standard wireless exponential decay model given in [21]. Suppose that the channel impulse response is composed of complex Gaussian samples with unit power, and the $l$-th delay path has an average power given by:

$$
\sigma_{l}^{2}=\left(1-e^{-1 / \tau}\right) e^{-l / \tau}
$$

where $\tau$ is the root-mean square (RMS) of the delay spread. Assume that the channel order $L=N_{g}=64$ is sufficiently large such that the impulse response tail can be neglected. The signal-to-noise ratio (SNR) is defined as $S N R=-10 \log _{10} \sigma_{N}^{2}$, where $\sigma_{N}^{2}$ is the average power of the noise. In the following simulations, the CFO search granularity used in Equations (9) and (16) is set to be $2^{-7}$ of the complete search interval. For the sake of comparison, the results of Chung's method [19] are also included while considering that the estimated channel order $\hat{L}$ is selected out of the set $\{2,16,62\}$. Without loss of generality, the parameter settings, including SNR $=30 \mathrm{~dB}, \tau=4, \epsilon_{d}=0.3$ and $J=5$, are used throughout this section.

The performance of the proposed CFO estimation is evaluated by the mean square error (MSE), which is expressed by:

$$
\operatorname{MSE}=\frac{1}{N_{s}} \sum_{i=1}^{N_{s}}\left|\hat{\epsilon}_{d}(i)-\epsilon_{d}\right|^{2}
$$

where $\epsilon_{d}$ is the actual CFO, $\hat{\epsilon}_{d}(i)$ is the estimated CFO obtained through the $i$-th trial and $N_{s}$ is the overall number of Monte Carlo trials. To investigate the MSE performance of the proposed iterative CFO estimation, in Figure 1, we demonstrate the convergence characteristic of the proposed iterative CFO estimation while assuming $\epsilon_{d}=0.3$. Although in Section 3, the iterative estimator is shown to cost much less complexity than the search-based scheme, the resultant expense is the reduction of the MSE convergence rate. When compared with the search-based scheme, the iterative method requires two more iterations to achieve the MSE convergence for all considered SNR values. It is noteworthy that at high SNR, say $40 \mathrm{~dB}$, the approximation error in Equation (30) cannot be neglected in relation to the noise effect. This is why the performance of the iterative scheme is somewhat inferior to the search-based estimation in the convergence performance. Figure 2 shows the MSE versus the iteration number for several specific CFO values. The figure indicates that the MSE using the iteration technique converges and approaches that for the search-based approach after three iterations when $\epsilon_{d}=0.4$. The larger the actual CFO is, the more iterations for achieving an estimate close to that of the proposed search-based scheme there will be. In Figure 3, we evaluate the MSE while considering certain RMSs of the channel delay spread. For $J>1$, it can be seen that the proposed iterative estimator provides distinguished robustness against the variation of the RMS of the delay spread and achieves a performance close to that of the search-based scheme. In addition, the MSE increases as the RMS becomes larger 
and larger, because a long delay spread will bring about a significant variation in the channel response between subcarriers.

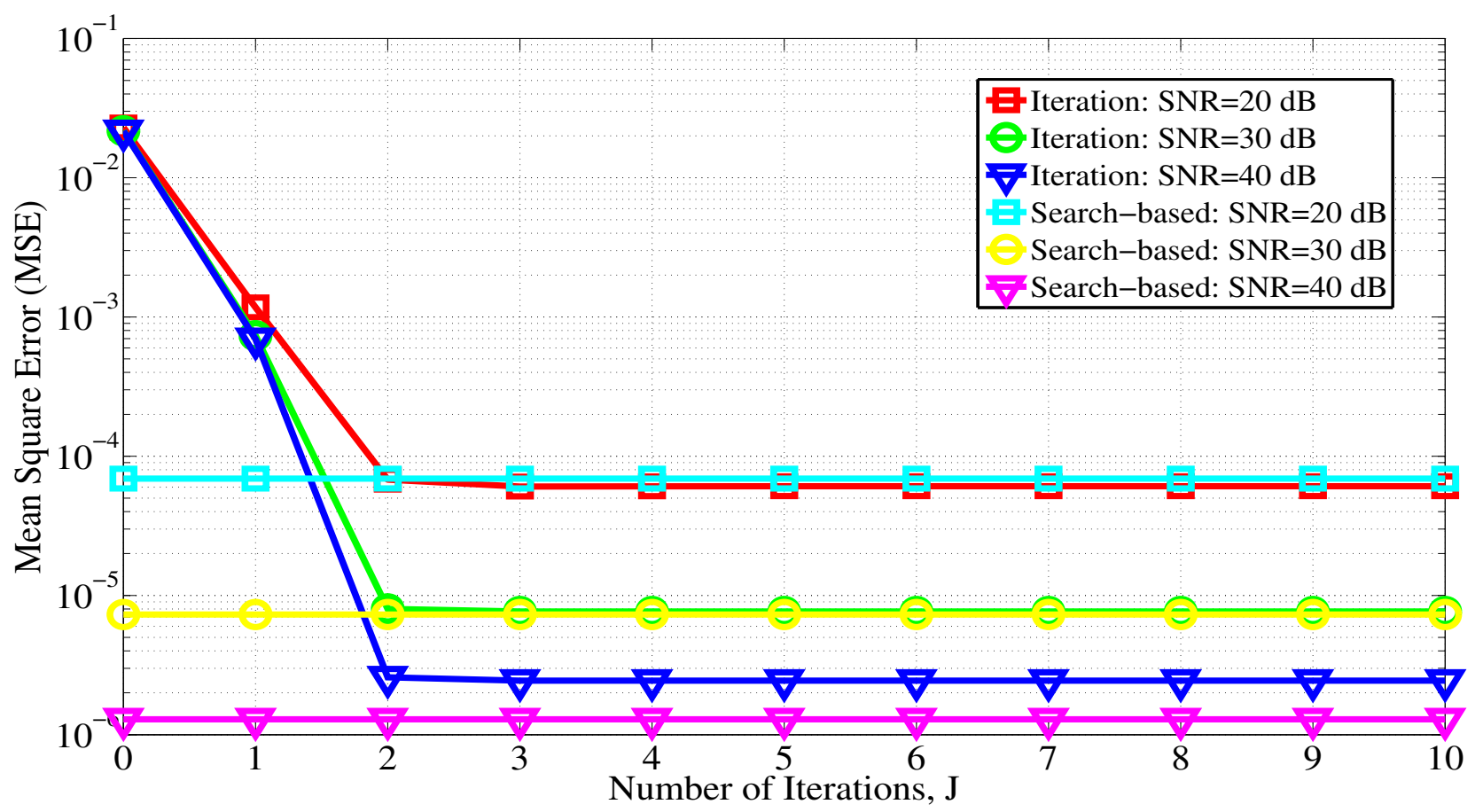

Figure 1. Convergence behavior of the proposed iterative CFO estimation for different SNRs. $N=256, K=64, \epsilon_{d}=0.3$ and $\tau=4$.

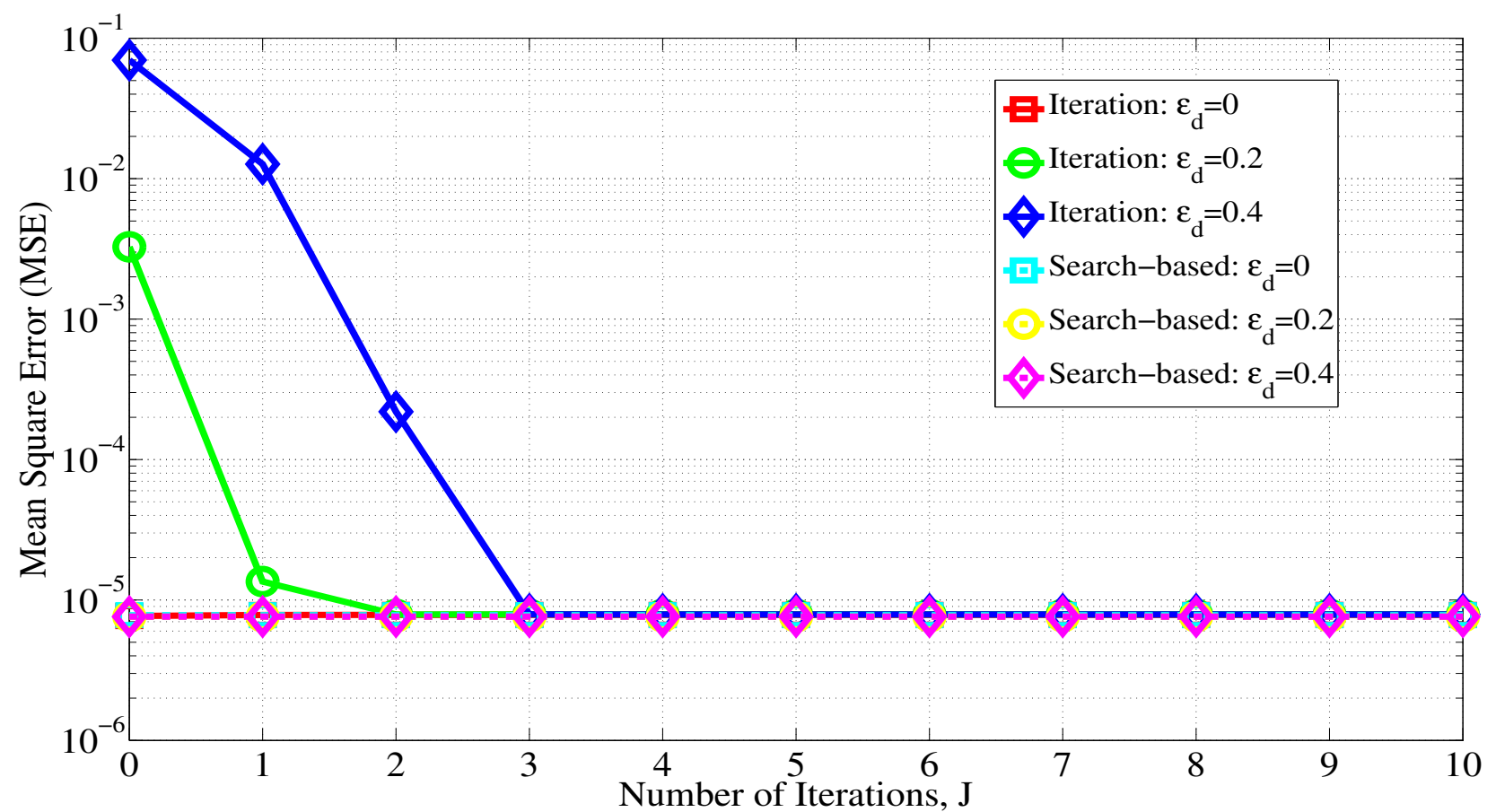

Figure 2. Convergence behavior of the proposed iterative CFO estimation for different CFOs. $N=256, K=64$, SNR $=30 \mathrm{~dB}$ and $\tau=4$. 


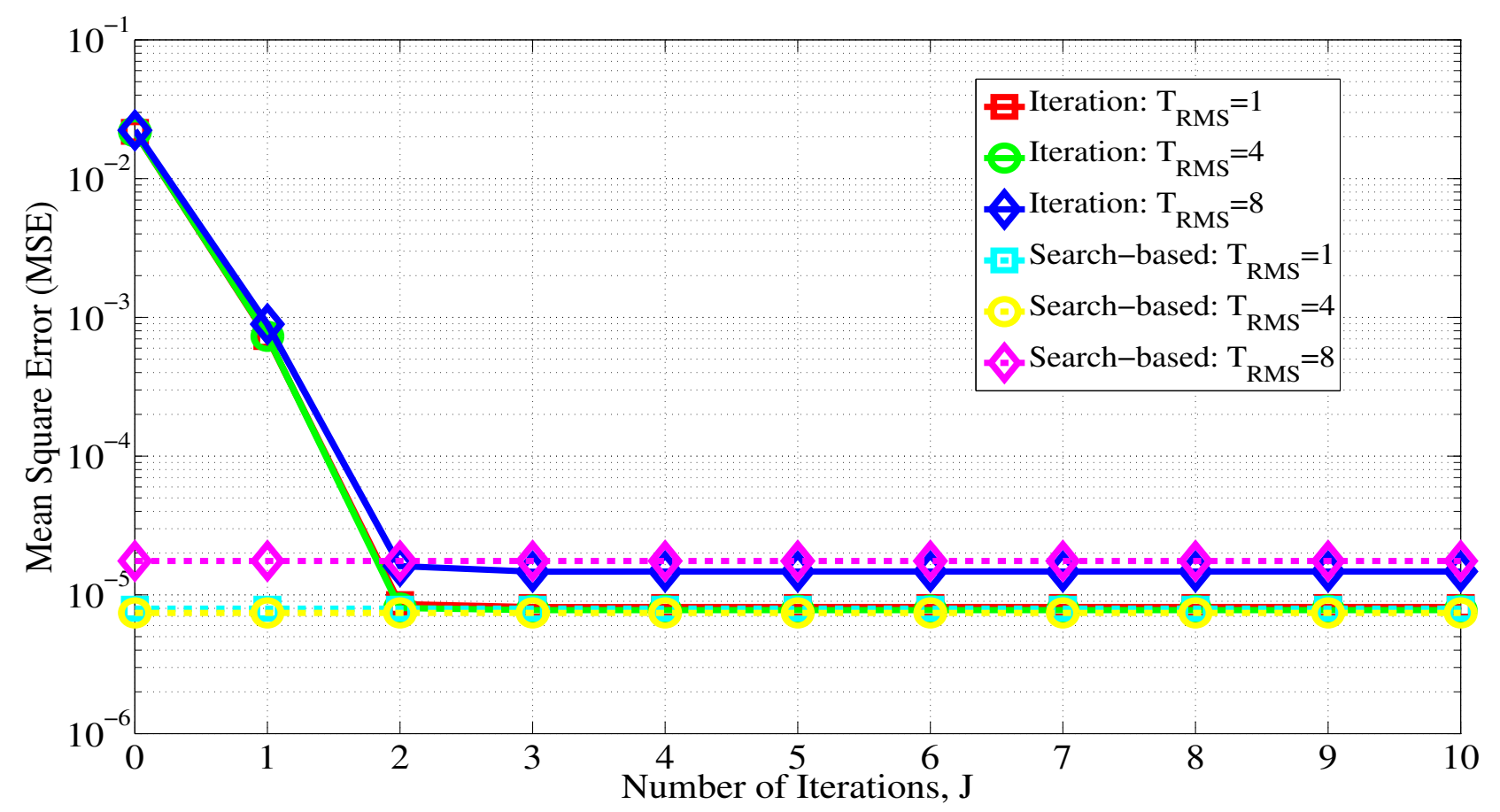

Figure 3. Convergence behavior of the proposed iterative CFO estimation for different RMSs of channel delay spread, $\tau . N=256, K=64, \mathrm{SNR}=30 \mathrm{~dB}$ and $\epsilon_{d}=0.3$.

In the next series of simulations, we compare the performance of the proposed two schemes with Chung's approach [19]. Figure 4 plots the MSE curves versus the input SNR when $\epsilon_{d}=0.3$ for all of the considered schemes. As expected, the MSE is shown to decrease as we increase the input SNR. The proposed iterative estimator requires five iterations to reach a performance comparable to our proposed search-based estimator while achieving the substantial reduction of computational complexity shown in Section 3. It can be found that Chung's method fails to offer a reliable estimate when the estimate of the channel order $L$ is not correctly provided. The MSE characteristics in response to the normalized CFO are presented in Figure 5 given $\mathrm{SNR}=30 \mathrm{~dB}$. It can be observed that the MSE performance is almost invariant to the actual CFO values regardless of the employed methods. However, in case an erroneous estimate on the channel order is made, there is significant performance degradation in the MSE of Chung's estimator. Figure 6 presents the MSE curves versus the RMS of the channel delay spread for all of the considered schemes. It shows that the MSE performances for both of the iterative and search-based estimators are nearly the same and gradually degrade while $T_{R M S}$ is getting larger. This is because the interpolated and actual channel responses do not coincide when the linear interpolation prevents the proposed schemes from acquiring a perfect time-domain channel response. The other way round is that Chung's method is strongly dependent on $T_{R M S}$. Especially, for the case with a large $T_{R M S}$, say $T_{R M S}>3$, the proposed schemes have much better performances than that using Chung's scheme. Note that there is a distinguished degradation in the MSE performance once again for using Chung's method when the channel response order $L$ is not well estimated in Figure 6. 


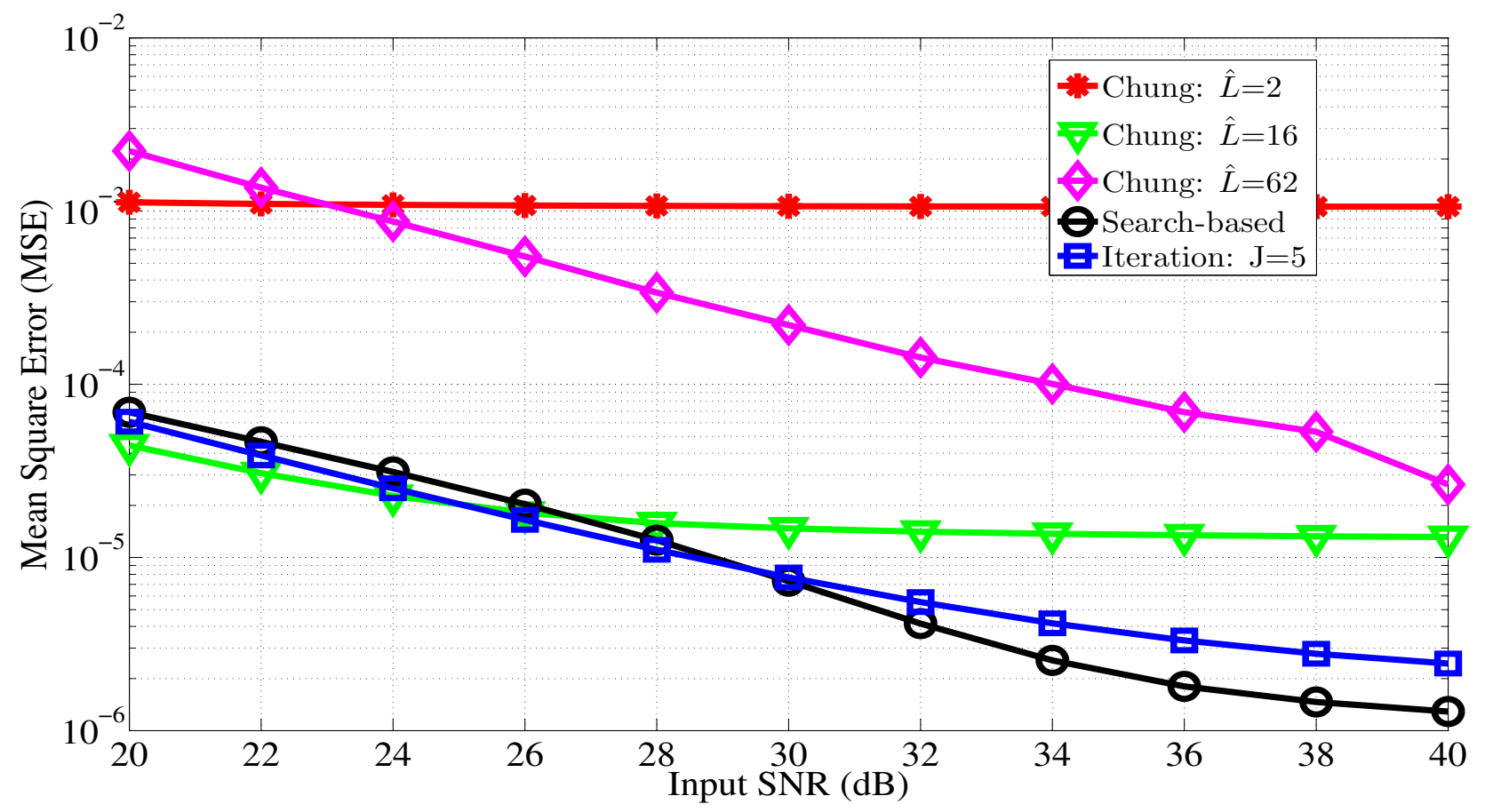

Figure 4. MSE performance of the proposed and Chung's CFO estimators for different SNRs. $N=256, K=64, \epsilon_{d}=0.3, \tau=4$ and $J=5$.

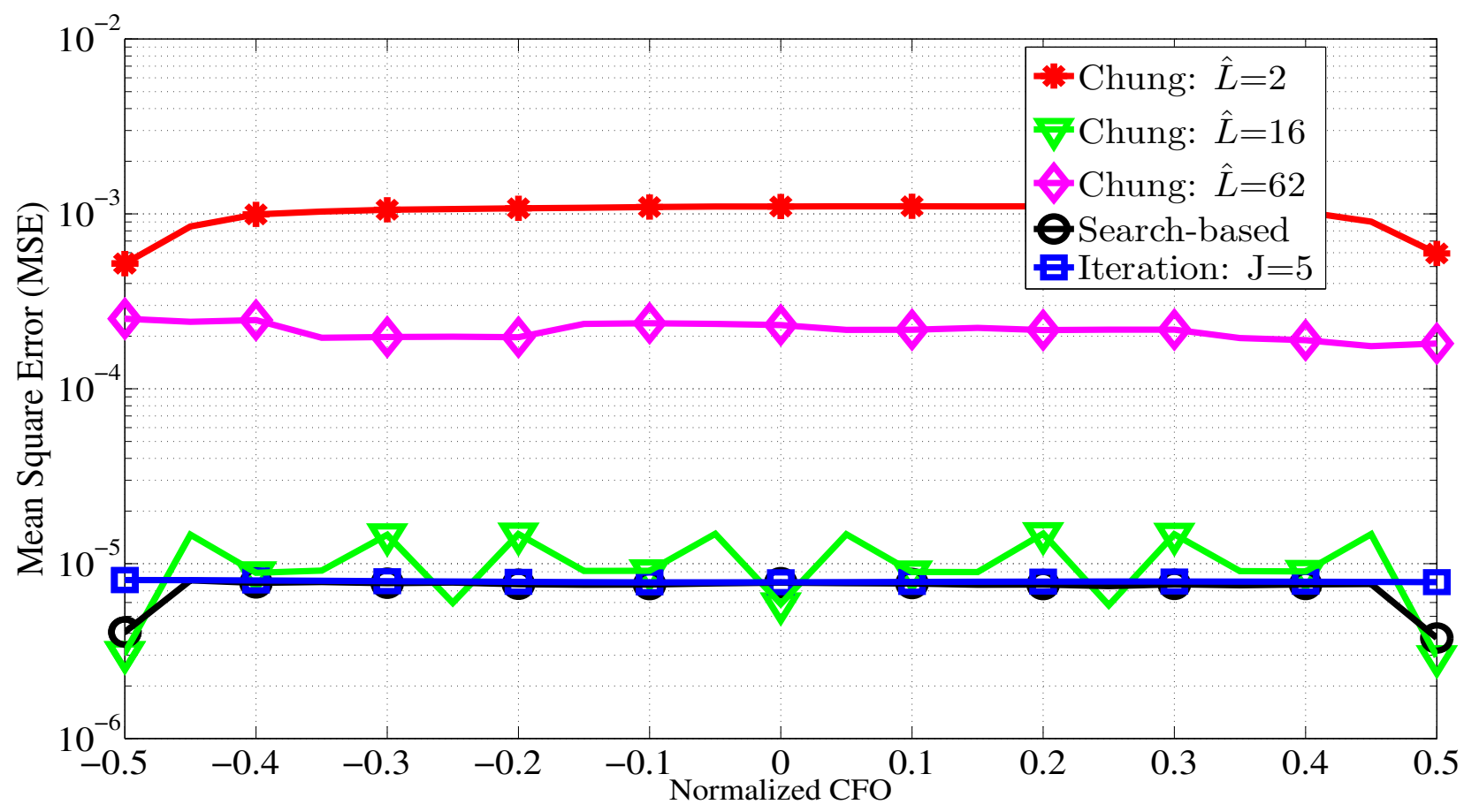

Figure 5. MSE performance of the proposed and Chung's CFO estimators for different CFOs. $N=256, K=64, \mathrm{SNR}=30 \mathrm{~dB}, \tau=4$ and $J=5$. 


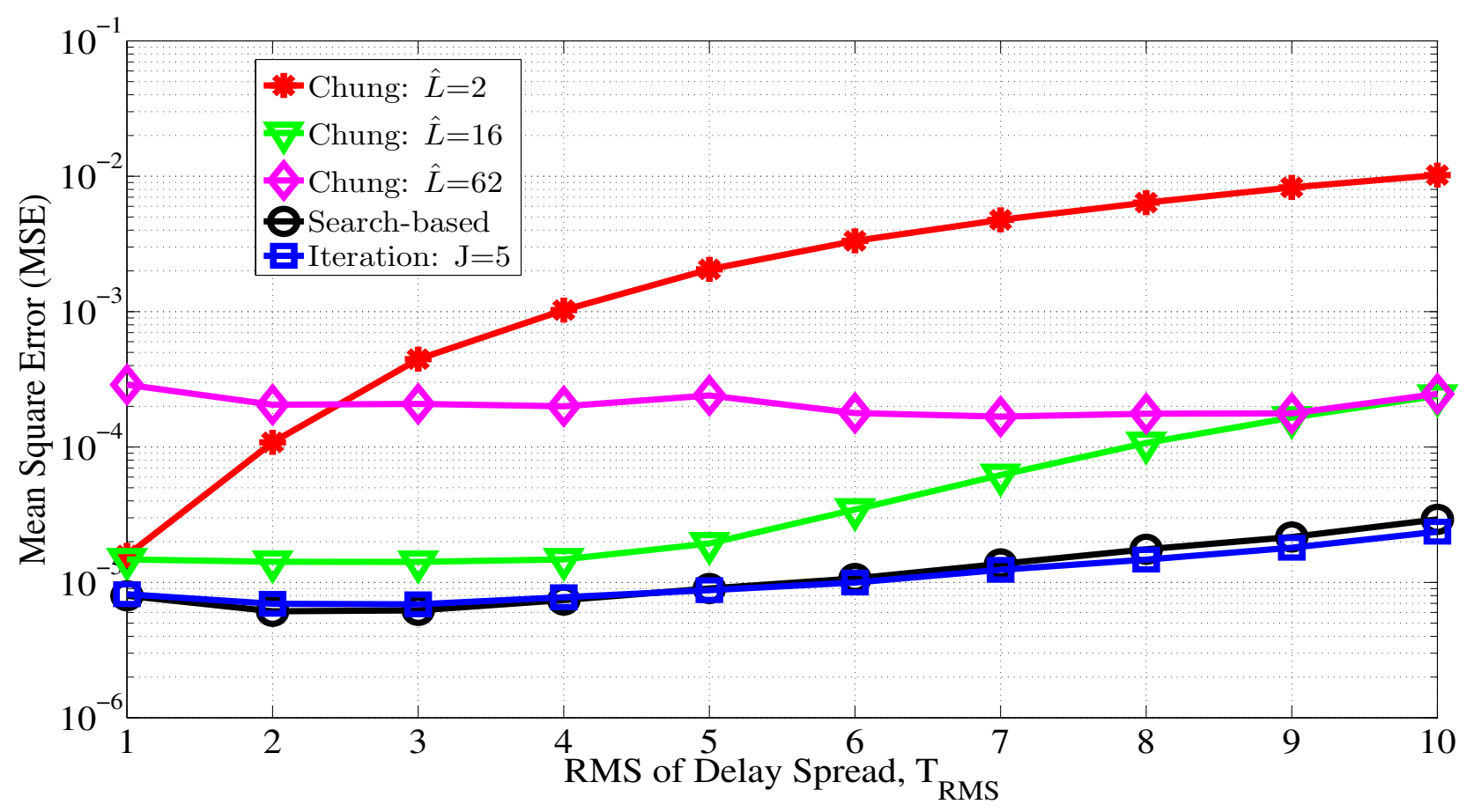

Figure 6. MSE performance of the proposed and Chung's CFO estimators for different RMSs of channel delay spread, $\tau . N=256, K=64, \mathrm{SNR}=30 \mathrm{~dB}, \epsilon_{d}=0.3$ and $J=5$.

\section{Conclusions}

Based on the analytical and simulation investigation presented in this paper, the conclusion may be made as follows. We have ameliorated the traditional search-based CFO estimator without using the prior knowledge of channel order. This contribution is made by exploiting an interpolation technique to reconstruct the channel frequency response, which is used to assist the CFO estimation algorithm. It is shown that not only the search-based, but also the iterative CFO estimators outperform Chung's method in the estimate performance. Besides, the numerical results verify that the employment of the iterative technique indeed reduces the estimator's complexity at the expense of the convergence rate of MSE. Fortunately, MSE will quickly converge after a couple of iterations. From the point of view of implementation complexity, the proposed schemes benefit principally from two factors. One is that we remove the limitation of knowledge of channel order, and the other is the replacement of the brute-force search. For realistic wireless applications, the proposed approaches appear to be more feasible than the conventional ones, such as Chung's method.

\section{Acknowledgments}

This work was supported in part by the Ministry of Science and Technology under MOST 104-2221-E-239-015. 


\section{Author Contributions}

Tsua-Tuai Lin and Fuh-Hsin Hwang designed and analyzed the proposed estimation technique. Tsua-Tuai Lin performed the computer simulations and analyzed the corresponding results. Tsua-Tuai Lin and Fuh-Hsin Hwang wrote the paper jointly.

\section{Conflicts of Interest}

The authors declare no conflict of interest.

\section{References}

1. Luise, M.; Reggiannini, R. Carrier frequency recovery in all-digital modems for burst-mode transmissions. IEEE Trans. Commun. 1995, 2-4, 1169-1178.

2. Tureli, U.; Liu, H.; Zoltowski, M.D. OFDM blind carrier offset estimation: ESPRIT. IEEE Trans. Commun. 2000, 9, 1459-1461.

3. Chen, D.; Xia, X.G.; Jiang, T.; Gao, X. Properties and Power Spectral Densities of CP Based OQAM-OFDM Systems. IEEE Trans. Signal Process. 2015, 7, 3561-3575.

4. Dai, L.; Wang, J.; Wang, Z.; Tsiaflakis, P.; Moonen, M. Spectrum- and energy-efficient OFDM based on simultaneous multi-channel reconstruction. IEEE Trans. Signal Process. 2013, 11, 6047-6059.

5. Chen, B. Maximum likelihood estimation of OFDM carrier frequency offset. IEEE Signal Process. Lett. 2002, 4, 123-126.

6. Huang, D.; Letaief, K.B. Carrier frequency offset estimation for OFDM systems using null subcarriers. IEEE Trans. Commun. 2006, 5, 813-823.

7. Hebley, M.G.; Taylor, D.P. The effect of diversity on a burstmode carrier-frequency estimator in the frequency-selective multipath channel. IEEE Trans. Commun. 1998, 4, 553-560.

8. Jeong, E.; Jo, S.; Lee, Y.H. Least square frequency estimation in frequency-selective channels and its application to transmissions with antenna diversity. IEEE J. Sel. Areas Commun. 2001, 12, 2369-2380.

9. Morelli, M.; Mengali, U. Carrier-frequency estimation for transmission over selective channels. IEEE Trans. Commun. 2000, 9, 1580-1589.

10. Moose, P.H. A technique for orthogonal frequency division multiplexing frequency offset correction. IEEE Trans. Commun. 1994, 10, 2908-2914.

11. Morelli, M.; Mengali, U. An improved frequency offset estimator for OFDM applications. IEEE Commun. Lett. 1999, 3, 75-77.

12. Morelli, M.; D'Andrea, A.N.; Mengali, U. Frequency ambiguity resolution in OFDM systems. IEEE Commun. Lett. 2000, 4, 134-136.

13. Wang, G.; Gao, F.; Wu, Y.C.; Tellambura, C. Joint CFO and channel estimation for OFDM-based two-way relay networks. IEEE Trans. Wireless Commun. 2011, 2, 456-465.

14. Huang, W.C.; Pan, C.H.; Li, C.P.; Li, H.J. Subspace-based semi-blind channel estimation in uplink OFDMA systems. IEEE Trans. Broadcast. 2010, 3, 58-65. 
15. Liang, Y.J.; Chang, J.F. Noniterative joint frequency offset and channel estimation for MIMO OFDM systems using cascaded orthogonal pilots. IEEE Trans. Veh. Technol. 2010, 10, 4151-4156.

16. Gao, F.; Zeng, Y.; Nallanathan, A.; Ng, T.S. Robust subspace blind channel estimation for cyclic prefix MIMO OFDM systems: Algorithm, identifiability and performance analysis. IEEE J. Sel. Areas Commun. 2008, 2, 378-388.

17. Van de Beek, J.J.; Sandell, M.; Borjesson, P.O. ML estimation of time and frequency offset in OFDM systems. IEEE Trans. Signal Process. 1997, 7, 1800-1805.

18. Wen, C.K.; Wang, Y.Y.; Chen, J.T. A low-complexity space-time OFDM multiuser system. IEEE Trans. Wirel. Commun. 2005, 5, 998-1007.

19. Chung, Y.H.; Phoong, S.M. OFDM channel estimation in the presence of receiver I/Q imbalance and CFO using pilot symbols. IEICE Trans. Commun. 2012, 2, 531-539.

20. Vaidyanathan, C.; Buckley, K.M. Performance analysis of the MVDR spatial spectrum estimator. IEEE Trans. Signal Process. 2012, 2, 531-539.

21. O'Hara, B.; Petrick, A. The IEEE 802.11 Handbook: A Designer's Compasion; IEEE Press: New York, NY, USA, 1999.

(C) 2015 by the authors; licensee MDPI, Basel, Switzerland. This article is an open access article distributed under the terms and conditions of the Creative Commons Attribution license (http://creativecommons.org/licenses/by/4.0/). 